\title{
Utility of brain CT for predicting delayed encephalopathy after acute carbon monoxide poisoning
}

\author{
XUQIN DU ${ }^{1}$, HUA GU $^{2}$, FENGTONG HAO ${ }^{1}$, LIN GAO $^{3}$, JIANRU WANG $^{4}$, CHENGYE SUN $^{5}$, \\ HONGSHUN ZHANG ${ }^{5}$, PEIBIN MA ${ }^{5}$, XINGGUO ZHANG ${ }^{6}$ and WENLI HU ${ }^{7}$
}

\author{
Departments of ${ }^{1}$ Occupational Medicine and Toxicology and ${ }^{2}$ Radiology, Beijing Chaoyang Hospital, \\ Capital Medical University, Beijing 100020; ${ }^{3}$ Shenyang Ninth People's Hospital, Shenyang, Liaoning 110024; \\ ${ }^{4}$ Shanxi Second People's Hospital, Taiyuan, Shanxi 030012; ${ }^{5}$ Chinese Center for Disease Control and Prevention, \\ Beijing 100050; ${ }^{6}$ Shandong Provincial Hospital, Jinan, Shandong 250021; ${ }^{7}$ Department of Neurology, \\ Beijing Chaoyang Hospital, Capital Medical University, Beijing 100020, P.R. China
}

Received May 7, 2018; Accepted January 11, 2019

DOI: 10.3892/etm.2019.7233

\begin{abstract}
Delayed encephalopathy after acute carbon monoxide poisoning (DEACMP) is a serious complication that occurs several days or weeks after carbon monoxide (CO) intoxication. This study identified computed tomography (CT) findings in the prediction of DEACMP development. Adults with CO poisoning admitted to Beijing Chaoyang Hospital, Shenyang Ninth People's Hospital, Shanxi Second People's Hospital and Shandong Provincial Hospital in China from January 2013 to January 2016 were retrospectively analyzed. Baseline demographic and clinical characteristics were extracted from the medical records. Brain CT imaging was carried out within $24 \mathrm{~h}$ of admission, and an integrated CT score was calculated to semi-quantify lesion severity. Patients were followed-up for 6 months. Baseline characteristics and $\mathrm{CT}$ findings were compared among patients who developed DEACMP (DEACMP group) and those who did not (non-DEACMP group). The receiver operating characteristic (ROC) curve analysis was used to examine the utility of integrated CT score for predicting DEACMP. Among the 123 patients included in the analysis, 27 (22.0\%) developed DEACMP. The DEACMP and non-DEACMP groups did not differ with regard to age, sex and carboxyhemoglobin $(\mathrm{COHb})$ level at admission. However, compared with the non-DEACMP group, the patients in the DEACMP group had longer onset time of symptoms and duration of exposure to $\mathrm{CO}$, higher acute physiology and chronic health evaluation-II (APACHE-II) score, lower Glasgow Coma Scale (GCS) score, and there was
\end{abstract}

Correspondence to: Dr Wenli Hu, Department of Neurology, Beijing Chaoyang Hospital, Capital Medical University, Beijing 100020, P.R. China

E-mail: heme9552@126.com

Key words: acute carbon monoxide poisoning, risk factor, delayed encephalopathy after acute carbon monoxide poisoning, computed tomography a higher proportion of patients with severe symptoms and brain $\mathrm{CT}$ abnormalities ( 81.5 vs. $51.0 \%$; $\mathrm{P}<0.05)$. Integrated CT score in the DEACMP group was significantly higher than that in the non-DEACMP group (73.63 vs. 51.39; $\mathrm{P}<0.01)$. ROC curve was used to analyze the utility of integrated CT score in the prediction of DEACMP. The area under the ROC curve was 0.700 (95\% confidence interval, 0.584-0.817; $\mathrm{P}<0.01$ ). In conclusion, brain integrated CT score has the potential to identify DEACMP in patients.

\section{Introduction}

Carbon monoxide (CO) is a colorless, odorless and tasteless gas harming human health at levels exceeding 100 ppm (1). Although CO poisoning is preventable, it remains an important cause of morbidity and mortality in modern society and a leading cause of death due to poising $(2,3)$. Common symptoms of $\mathrm{CO}$ poisoning include headache, nausea, vomiting, dizziness, malaise and altered mental state as well as myocardial ischemia, chest pain and shortness of breath in some cases (4). The toxicity of $\mathrm{CO}$ is mainly caused by the high affinity of $\mathrm{CO}$ for hemoglobin, which results in tissue hypoxia (1). In addition, various cellular mechanisms have been implicated in the toxic effects of $\mathrm{CO}$, including binding of $\mathrm{CO}$ to other intracellular proteins (such as myoglobin and cytochrome), generation of nitric oxide and peroxynitrite, lipid peroxidation, mitochondrial oxidative stress, immune-mediated damage and apoptosis (2). Administration of oxygen is the main treatment of $\mathrm{CO}$ poisoning, which can accelerate the elimination of carboxyhemoglobin $(\mathrm{COHb})$ and reduce tissue hypoxia.

Several studies have reported that hyperbaric oxygen therapy is an effective treatment for CO poisoning (4-6). Furthermore, the addition of dexamethasone (7) or $N$-butylphthalide (8) to hyperbaric oxygen therapy may also exert beneficial effects.

Delayed encephalopathy after acute carbon monoxide poisoning (DEACMP) is a serious and common complication of exposure to $\mathrm{CO}$ at toxic levels $(9,10)$. DEACMP manifests as memory impairment, cognitive dysfunction and behavioral disorders that develop several days or weeks after 
acute exposure to $\mathrm{CO}(9,10)$. There is some evidence that hyperbaric oxygen therapy may be beneficial in patients with DEACMP (11). Nonetheless, the prognosis of DEACMP is poor, and the factors associated with clinical outcomes include age, lucid interval (between the initial recovery from the acute CO intoxication and the subsequent appearance of DEACMP symptoms), danger activities of daily living score, complications (11) and diffusion tensor imaging findings (12).

Identifying patients with acute $\mathrm{CO}$ poisoning that would go on to develop DEACMP would facilitate decision-making regarding treatment strategies, but this identification is challenging. Reported risk factors for DEACMP include age $>35$ years, $\mathrm{CO}$ exposure for $>24 \mathrm{~h}$, serious disturbance of consciousness at emergency admission, prolonged disturbance of consciousness, elevated levels of creatine kinase, creatine kinase-MB and lactate dehydrogenase, and low Global Assessment Scale score (13-16). However, the clinical utility of these factors in predicting DEACMP is limited. Magnetic resonance imaging (MRI) has revealed that DEACMP is associated with multiple lesions in the basal ganglia (mainly the globus pallidus, but also in the caudate nucleus and putamen alone), subcortical and periventricular white matter, cerebral cortex and hippocampus (17-19). In light of this, it is valuable to apply imaging methods to identify patients at risk of developing DEACMP. Although MRI can identify cerebral lesions in patients with acute $\mathrm{CO}$ poisoning, one study has found that the presence of MRI lesions at 1 month after $\mathrm{CO}$ intoxication was unable to accurately predict longer-term clinical outcome (20). Computed tomography (CT) is a clinically routine and non-invasive imaging technique that may have the potential to identify patients at risk of DEACMP. Two previous studies have reported that CT findings of hypoxic encephalopathy are related to the development of DEACMP $(16,21)$. However, there remains a paucity of data regarding the utility of CT for identifying patients at risk of DEACMP.

This study identified the early-stage clinical features and CT findings that predicted the development of DEACMP in patients with acute $\mathrm{CO}$ poisoning.

\section{Patients and methods}

Study design and patients. Patients with acute $\mathrm{CO}$ poisoning admitted to Beijing Chaoyang Hospital, Shenyang Ninth People's Hospital, Shanxi Second People's Hospital and Shandong Provincial Hospital in China from January 2013 to January 2016 were retrospectively analyzed. Inclusion criteria: i) patients aged 18-75 years; ii) those diagnosed with acute $\mathrm{CO}$ poisoning based on signs, symptoms and $\mathrm{COHb}$ levels $(22,23)$; iii) those imaged by brain CT during the early stages (i.e., within $24 \mathrm{~h}$ of admission to hospital); and iv) those who were diagnosed or suspected of the disease before the development of DEACMP. Exclusion criteria: i) patients with history of severe cerebrovascular disease, epilepsy, encephalitis, meningitis or congenital neurologic disease; ii) those with history of severe heart, liver or kidney disease; iii) those who had visited several hospitals, been ill for several days (with obvious signs and symptoms), or not received a CT examination at an early stage; and iv) women who were pregnant or breastfeeding. This study was approved by the Ethics Committee of the China Center for
Disease Control and Prevention (Beijing, China), but patients did not sign the informed consent due to the retrospective design of the study.

Diagnosis of DEACMP. DEACMP was diagnosed if any of the following clinical abnormalities were observed at 2-60 days after the initial recovery from the consciousness disorder caused by acute $\mathrm{CO}$ poisoning: i) disturbance of mental state and/or consciousness manifesting as dementia or delirium; ii) extrapyramidal neuropathy manifesting as Parkinson's syndrome; iii) pyramidal neurologic damage (such as hemiplegia, pathologic reflexes or urinary incontinence); and iv) focal cerebral cortical dysfunction (such as aphasia, blindness or the occurrence of secondary epilepsy). Additional evidence of DEACMP included pathologic low-density regions in brain CT images and moderate or severe abnormalities in the electroencephalogram.

Brain CT imaging. Brain CT imaging was performed immediately and was repeated within $24 \mathrm{~h}$ after the occurrence or suspected occurrence of toxic encephalopathy symptoms (to aid in the diagnosis of DEACMP). The CT scanner used was either a SOMATOM Emotion 16 (Siemens Healthineers, Erlangen, Germany) or a BrightSpeed Elite (GE Healthcare, Chicago, IL, USA), and the scanning parameters were: layer thickness, $5 \mathrm{~mm}$; layer interval, $10 \mathrm{~mm}$.

Analysis of CT images. The CT value within a region of interest $\left(2-5 \mathrm{~cm}^{2}\right.$, adjusted according to the size of the site being analyzed) was measured in the following brain regions: bilateral centrum semiovale, white matter of the frontal, parietal, occipital and temporal lobes, basal ganglia, thalamus, capsula interna, cerebral peduncle, pons and cerebellum (mainly white matter). Except for special cases, when the original data could not be provided and the CT value was measured only by the doctor appointed by the local hospital, all CT values were measured independently by two senior radiologists (with 22 and 26 years of work experience, respectively), with each radiologist measuring the CT value of each brain region 3 times to obtain an average value.

Semi-quantitative analysis of the CT abnormalities. Three experienced radiologists (each with $>20$ years of work experience) independently calculated an integrated CT score, based on the characteristics of the regions of abnormal density, and an average value was obtained for each patient. This approach is not subject to the limitations of the CT scanner, allowing the data to be directly compared between hospitals. The method used to determine scores was as follows: i) distribution of lesion for each brain region: 0 , no abnormal density; +1 , unilateral density abnormality; +2 , bilateral density abnormality. The scores for the various brain regions analyzed were summated to provide an overall score for lesion distribution; a higher score indicated a wider distribution of abnormal density. ii) Extent of lesion: +1, when the density/abnormality was limited to the deep white matter; +2 , when the density/abnormality was both in deep white matter and in subcortical white matter. iii) Lesion severity: +1 , when the reduction of CT value was $\leq 2-5$ Hounsfield units (HU); +2 , when the reduction in $\mathrm{CT}$ value was $>5 \mathrm{HU}$. The scores 
Table I. Baseline demographic and clinical characteristics of the study participants.

\begin{tabular}{lccr}
\hline Characteristics & $\begin{array}{c}\text { DEACMP group } \\
(\mathrm{n}=27)\end{array}$ & $\begin{array}{c}\text { Non-DEACMP group } \\
(\mathrm{n}=96)\end{array}$ & $\begin{array}{r}\text { P-value } \\
\hline \text { Sex }\end{array}$ \\
Male & $19(70.4 \%)$ & $54(56.2 \%)$ & 0.187 \\
Female & $8(29.6 \%)$ & $42(43.8 \%)$ & 0.403 \\
Age (years) & $48.15 \pm 13.25$ & $45.23 \pm 16.64$ & 0.020 \\
Onset time of symptoms (h) & 75.57 & 58.18 & $<0.001$ \\
Duration of exposure to CO (h) & $13.11 \pm 7.26$ & $8.12 \pm 4.71$ & 0.002 \\
Abnormal cranial CT findings at admission & $22(81.5 \%)$ & $49(51.0 \%)$ & 0.325 \\
COHb level at admission & $27.10 \pm 12.32 \%$ & $24.36 \pm 12.85 \%$ & $<0.001$ \\
APACHE-II score & $16.48 \pm 5.25$ & $9.02 \pm 5.22$ & $<0.001$ \\
GCS score & $4.59 \pm 1.76$ & $9.28 \pm 4.10$ & 0.001 \\
Severity of clinical symptoms & & & \\
Mild & $3(11.1 \%)$ & $49(51.0 \%)$ & $34(35.4 \%)$ \\
Moderate & $16(59.3 \%)$ & $13(13.5 \%)$ & \\
Severe & $8(29.6 \%)$ & & \\
\hline
\end{tabular}

Statistical comparisons were made using the independent samples t-test or Chi-square test (normal distribution), and the rank sum test (non-normal distribution). Mean rank values are shown. DEACMP, delayed encephalopathy after acute carbon monoxide poisoning; CO, carbon monoxide; CT, computed tomography; COHb, carboxyhemoglobin; APACHE-II, acute physiology and chronic health evaluation-II; GCS, Glasgow Coma Scale.

for various brain regions were summarized to provide an overall score for lesion severity. iv) Swelling of parenchyma: 0 , no brain swelling; +1 , sulcal effacement; +2 , ventricle is subjected to compression and becomes narrower. v) Other, including cerebral cortex involvement, hemorrhagic lesion and hippocampal lesion: +1 , one condition; +2 , two conditions; +3 , $\geq 3$ conditions. The above scores were summarized to give a final total score for each patient; a higher total score was taken to indicate more serious disease.

Fig. 1 shows an example of semi-quantitative analysis of the CT abnormalities. The patient was diagnosed with acute $\mathrm{CO}$ poisoning and the brain CT was at onset (within $24 \mathrm{~h}$ ). Distribution of lesion: +2 , the areas of subcortical decreased attenuation in bilateral frontal (arrows a and b) and occipital lobe (arrows e and f); +2 , bilateral globus pallidus (arrows c and d). Extent of lesion: +1 , deep white matter, no subcortical white matter involvement, frontal and occipital lobe. Lesion severity: +1 , hypodensity of parenchyma, CT value $\leq 2-5 \mathrm{HU}$, frontal lobe, occipital lobe and globus pallidus. Swelling of parenchyma: +1 , sulcal effacement; +0 , no involvement of other regions. The total integrated CT score of the patient was 11 .

Data collection. The following information was extracted from the patients' medical records: age, sex, onset time of symptoms, duration of exposure to $\mathrm{CO}, \mathrm{COHb}$ level at admission, acute physiology and chronic health evaluation-II (APACHE-II) scale score (24), Glasgow Coma Scale (GCS) score (25) and abnormal CT findings. Besides, the severity of the clinical symptoms was classified as mild, moderate or severe based on the APACHE-II score and the

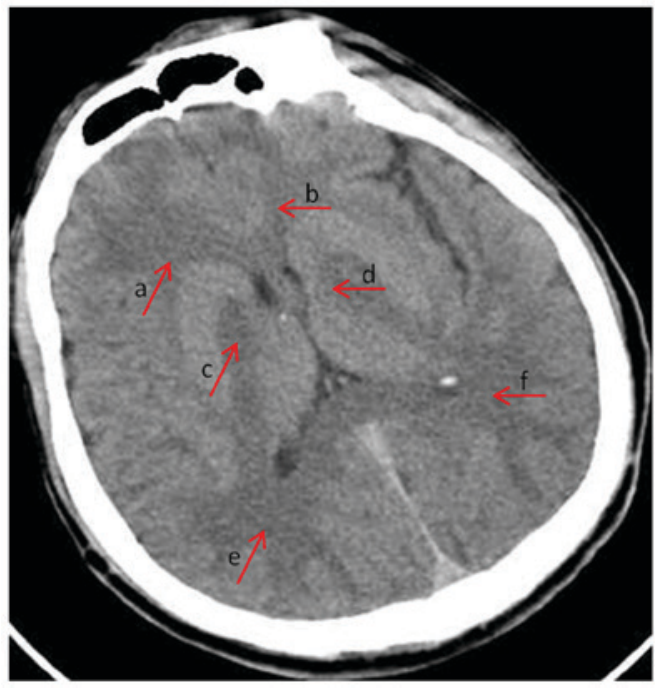

Figure 1. Semi-quantitative analysis of CT abnormalities. Figure shows the distribution of lesion: +2 , the areas of subcortical decreased attenuation in bilateral frontal (arrows a and b) and occipital lobe (arrows e and f); +2 , bilateral globus pallidus (arrows c and d).

diagnosis of $\mathrm{CO}$ poisoning based on signs, symptoms and COHb levels $(22,23)$.

Follow-up. Each patient was followed-up for 6 months from the occurrence of acute $\mathrm{CO}$ poisoning and was reviewed daily for the first 2 months and then monthly for the remaining period. Follow-up was conducted in the hospital or clinic. GCS score, APACHE-II score and brain CT images were evaluated 
Table II. Comparison of CT values of various brain regions among patients who developed delayed encephalopathy and those who did not.

\begin{tabular}{|c|c|c|c|}
\hline Brain region & DEACMP group & Non-DEACMP group & P-value \\
\hline Siemens Healthineers scanner & 18 & 51 & \\
\hline Centrum semiovale (HU) & $27.43 \pm 2.82$ & $28.12 \pm 2.56$ & 0.205 \\
\hline Frontal lobe (HU) & $28.37 \pm 3.77$ & $29.23 \pm 5.29$ & 0.386 \\
\hline Parietal lobe (HU) & $27.86 \pm 4.06$ & $28.83 \pm 5.12$ & 0.409 \\
\hline Occipital lobe (HU) & $28.09 \pm 3.79$ & $29.67 \pm 4.99$ & 0.167 \\
\hline Temporal lobe (HU) & $31.80 \pm 4.28$ & $31.51 \pm 4.29$ & 0.719 \\
\hline Globus pallidus (HU) & $29.93 \pm 3.29$ & $31.98 \pm 3.70$ & 0.014 \\
\hline Thalamus (HU) & $32.68 \pm 2.06$ & $33.82 \pm 3.13$ & 0.074 \\
\hline Capsula interna (HU) & $31.28 \pm 4.79$ & $30.02 \pm 4.55$ & 0.291 \\
\hline Cerebral peduncle (HU) & $30.15 \pm 2.30$ & $30.49 \pm 2.84$ & 0.618 \\
\hline Pons (HU) & $31.49 \pm 4.35$ & $31.39 \pm 4.25$ & 0.930 \\
\hline Cerebellar vermis (HU) & $36.19 \pm 2.63$ & $36.76 \pm 3.82$ & 0.521 \\
\hline Cerebellar dentate nucleus (HU) & $30.42 \pm 4.18$ & $31.69 \pm 3.74$ & 0.578 \\
\hline GE Healthcare scanner, $\mathrm{n}$ & 9 & 45 & \\
\hline Centrum semiovale (HU) & $23.21 \pm 2.34$ & $22.72 \pm 3.04$ & 0.951 \\
\hline Frontal lobe (HU) & $23.48 \pm 3.03$ & $24.03 \pm 2.85$ & 0.717 \\
\hline Parietal lobe (HU) & $23.64 \pm 2.12$ & $23.64 \pm 3.02$ & 0.997 \\
\hline Occipital lobe (HU) & $24.77 \pm 2.64$ & $23.94 \pm 3.06$ & 0.559 \\
\hline Temporal lobe (HU) & $23.48 \pm 3.37$ & $24.02 \pm 3.35$ & 0.717 \\
\hline Globus pallidus (HU) & $29.67 \pm 2.45$ & $28.43 \pm 3.94$ & 0.544 \\
\hline Thalamus (HU) & $30.93 \pm 4.31$ & $30.58 \pm 2.74$ & 0.837 \\
\hline Capsula interna (HU) & $22.67 \pm 4.16$ & $23.68 \pm 3.40$ & 0.625 \\
\hline Cerebral peduncle (HU) & $26.07 \pm 3.30$ & $26.60 \pm 3.18$ & 0.781 \\
\hline Pons (HU) & $27.63 \pm 3.51$ & $26.93 \pm 2.66$ & 0.668 \\
\hline Cerebellar vermis (HU) & $31.50 \pm 3.05$ & $33.24 \pm 3.74$ & 0.467 \\
\hline Cerebellar dentate nucleus (HU) & $27.05 \pm 5.46$ & $26.84 \pm 4.08$ & 0.925 \\
\hline
\end{tabular}

Statistical comparisons were made using the independent samples t-test. CT values are expressed as mean \pm SD. CT, computed tomography; DEACMP, delayed encephalopathy after acute carbon monoxide poisoning; HU, Hounsfield units.

at onset. The occurrence of DEACMP was determined on the basis of the patient's clinical symptoms and signs (vide supra).

Statistical analysis. Statistical analysis was performed using SPSS for Windows, release 19.0 (IBM Corp., Armonk, NY, USA). Data are presented as the mean \pm standard deviation (SD) or $\mathrm{n}(\%)$ and compared using the independent samples t-test, Chi-square test or rank sum test, as appropriate. Non-normally distributed data were compared with the rank sum test. The utility of the integrated CT value in the prediction of DEACMP was explored using the receiver operating characteristic (ROC) curve analysis with calculation of the area under the curve (AUC). $\mathrm{P}<0.05$ was considered to indicate a statistically significant difference.

\section{Results}

Baseline demographic and clinical characteristics. A total of 123 patients with acute $\mathrm{CO}$ poisoning were included in the analysis, among whom 27 (22.0\%) developed DEACMP (DEACMP group) and 96 (78.0\%) did not (non-DEACMP group). The baseline demographic and clinical characteristics of the study participants are presented in Table I. There were no significant differences between the DEACMP and non-DEACMP group in age, sex, and $\mathrm{COHb}$ level at admission (Table I). However, compared with patients in the non-DEACMP group, those in the DEACMP group had a longer duration of exposure to $\mathrm{CO}(13.11 \pm 7.26 \mathrm{vs} .8 .12 \pm 4.71 \mathrm{~h}$; $\mathrm{P}<0.001)$, higher APACHE-II score $(16.48 \pm 5.25$ vs. 9.02 \pm 5.22 ;

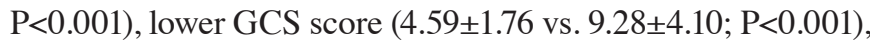
and there was a higher proportion of patients with severe clinical symptoms $(\mathrm{P}=0.001$; Table $\mathrm{I})$ and brain $\mathrm{CT}$ abnormalities (81.5 vs. $51.0 \%$; $\mathrm{P}=0.002)$.

Comparison of CT values between the DEACMP and the non-DEACMP group. As shown in Table II, the CT values were compared between the DEACMP and non-DEACMP group for 69 patients scanned with the Siemens SOMATOM Emotion 16 scanner and 54 patients scanned with the GE Healthcare scanner. For patients scanned with the Siemens CT scanner, there were no significant differences in the CT values of the various brain regions, with the exception of the globus 
Table III. Comparison of integrated CT scores among patients who developed delayed encephalopathy and those who did not.

\begin{tabular}{lccr}
\hline Brain region & $\begin{array}{c}\text { DEACMP group } \\
(\mathrm{n}=23)\end{array}$ & $\begin{array}{c}\text { Non-DEACMP group } \\
(\mathrm{n}=88)\end{array}$ & P-value \\
\hline Basal ganglia & $8.69(\mathrm{n}=8)$ & $10.95(\mathrm{n}=11)$ & 0.354 \\
White matter & $36.00(\mathrm{n}=18)$ & $32.56(\mathrm{n}=48)$ & 0.477 \\
Other regions & $5.00(\mathrm{n}=2)$ & $5.62(\mathrm{n}=8)$ & 0.788 \\
Total score & $73.63(\mathrm{n}=23)$ & $51.39(\mathrm{n}=88)$ & 0.002 \\
\hline
\end{tabular}

Statistical comparisons were made using the rank sum test (non-normal distribution). Mean rank values are shown. CT, computed tomography; DEACMP, delayed encephalopathy after acute carbon monoxide poisoning.

pallidus, which had a lower CT value in the DEACMP group than in the non-DEACMP group $(29.93 \pm 3.29$ vs. $31.98 \pm 3.70$; $\mathrm{P}=0.014$; Table II). For patients scanned with the GE Healthcare scanner, there were no significant differences in the CT values of any of the brain regions between the two groups (Table II).

Comparison of integrated CT scores between the DEACMP and non-DEACMP group. Integrated CT scores were calculated for 111 out of the 123 patients (the original images could not be obtained in 12 patients for the determination of the integrated CT scores). As shown in Table III, the DEACMP and non-DEACMP group did not differ significantly with regard to the integrated $\mathrm{CT}$ scores of the basal ganglia, white matter or other regions when these brain regions were considered individually. However, the total integrated CT score in the DEACMP group was notably higher than that in the non-DEACMP group (73.63 vs. 51.39; $\mathrm{P}=0.002)$.

ROC curve analysis of the utility of integrated CT score in the prediction of DEACMP. The area under the ROC curve was 0.700 , with a $95 \%$ confidence interval of $0.584-0.817(\mathrm{P}<0.01)$.

\section{Discussion}

An important finding of the present study was that the DEACMP group had a longer duration of $\mathrm{CO}$ exposure, higher APACHE-II score, lower GCS score, greater symptom severity and higher proportion of patients with brain CT abnormalities. Furthermore, the $\mathrm{CT}$ value in various brain regions could not be used to distinguish between patients who subsequently developed DEACMP and those who did not. Importantly, integrated CT score was significantly higher in the DEACMP group than in the non-DEACMP group and ROC curve analysis indicated that total integrated CT score could potentially be used as a predictor of DEACMP. Our novel findings indicate that total integrated CT score could potentially be used to identify DEACMP in patients.

In this study, DEACMP subsequently occurred in $22 \%$ of those with acute $\mathrm{CO}$ poisoning, consistent with previous investigations reporting values ranging from 16.5 to $29.5 \%(14,15,21)$. No difference was found in sex between the two groups of patients in this study, in consistency with a previous publication (15). Age of patients has been proven to influence the incidence rate of DEACMP, although there are inconsistencies among the published data.
For example, Choi has found that DEACMP does not occur in patients aged $<30$ years (16), while Weaver et al have determined that at age $>35$ years the risk in patients not treated with hyperbaric oxygen is increased but the risk in those that have received hyperbaric oxygen therapy is decreased (14). In agreement with our findings, Kudo et al observed no difference in age between patients that developed DEACMP and those that did not (15).

In this study, disease severity, regardless of the score used (APACHE-II score, GCS score or clinical symptom score), was markedly higher in the DEACMP group than that in the non-DEACMP group, which is consistent with the findings of Kudo et al that patients who suffered from DEACMP had a significantly higher Japan Coma Scale score (15). Other studies have also suggested that impaired consciousness is correlated with DEACMP (16). It would perhaps be expected that the degree of exposure to $\mathrm{CO}$ would influence the severity of the CO poisoning and hence the risk of DEACMP. It was observed that patients in the DEACMP group had a longer duration of exposure to $\mathrm{CO}$ than those in the non-DEACAMP group, in agreement with a previous report that exposure for $24 \mathrm{~h}$ or longer is an independent risk factor for cognitive sequelae (14). Although another study has found no influence of duration of CO exposure on risk of DEACMP (15), relevant data were available for only half of their cohort of patients, and the authors noted that variations in individual circumstances (particularly size and ventilation of the location of exposure) could have affected the interpretation of the results. $\mathrm{COHb}$ at first hospital consultation did not appear to be associated with the development of DEACMP in our study or clinical outcomes in other published research $(15,26)$. The lack of an association of $\mathrm{COHb}$ at first hospital consultation with DEACMP may be due to the relatively short half-life of $\mathrm{COHb}$ and hence the rapid fall in $\mathrm{COHb}$ level that occurs after cessation of $\mathrm{CO}$ inhalation and administration of hyperbaric oxygen.

There is very little research exploring whether CT imaging could be used to predict the risk of DEACMP. An important finding of the present study was that the DEACMP group had a higher proportion of patients with brain CT abnormalities (81.5 vs. 51.0\%). Similarly, Choi et al have determined that $62.2 \%$ of the patients that developed delayed neurologic sequelae had initial CT abnormalities, compared with only $27.0 \%$ of patients that made a full recovery (21). Kudo et al have also found that their DEACMP group had substantially more patients with brain CT abnormalities than their non-DEACMP 
group (76.9 vs. 6.2\%) (15). However, in this investigation, CT values in the various brain regions examined did not differ between groups, indicating that this parameter may not be useful for predicting the risk of DEACMP. In contrast, the integrated CT score differed significantly between patients who developed DEACMP and those who did not, and ROC curve analysis suggested that this parameter could potentially be used to predict the risk of DEACMP. An important advantage of integrated CT score is that it is independent of the scanner characteristics, allowing it to be used widely in different Institutions. The integrated CT score is related to the severity of the patient's lesions and thus is an indirect evaluation of the pathologic changes in the brain. Therefore, this parameter may be a novel approach to risk stratification in patients with acute $\mathrm{CO}$ poisoning.

This study has some limitations. It was a retrospective study, so it might have been prone to information bias and selection bias. Acute $\mathrm{CO}$ poisoning is relatively rare, hence the sample size was small despite the inclusion of four study sites. Therefore, the results could not be generalized to other regions of China or other countries. Unknown confounding factors that were not included in the analysis might have influenced the results. Direct comparisons of CT with other imaging modalities, such as DWIMRI, were not made. The most commonly used quantitative indicators of DWI are average diffusion coefficient (ADC) and fractional nisotropy (FA). In our study, DWI findings were not involved. The most commonly used quantitative indicators of DWI are ADC and FA. The associations between CT findings and long-term clinical outcomes were not examined. Additionally, prospective, multicenter, large-scale studies are merited to further explore the utility of $\mathrm{CT}$ in predicting the risk of DEACMP. In conclusion, brain integrated CT score can potentially identify patients at risk of DEACMP.

\section{Acknowledgements}

We would like to thank all patients and investigators who were involved in this study. We also express our thanks to Dr Jinsong Zhang and Dr Haishi Wang for their advice on data analysis and determination of CT values and scores.

\section{Funding}

The study was supported by the Special Project for Scientific Research in the Health Industry of China (201202006-06).

\section{Availability of data and materials}

The datasets used and/or analyzed during the current study are available from the corresponding author on reasonable request.

\section{Authors' contributions}

$\mathrm{XD}$ and $\mathrm{WH}$ contributed in the data analysis and were involved in the design of the study. XD, HG and FH were responsible for determining the $\mathrm{CT}$ values and calculating the integrated CT scores. LG, JW and XZ participated in the data acquisition and the follow-up management of the patients. CS, HZ and PM were involved in the design of the methods and the interpretation of the data. All authors read and approved the final manuscript.

\section{Ethics approval and consent to participate}

The study was approved by the Ethics Committee of Beijing Chaoyang Hospital (Beijing, China) and the Ethics Committee of the Chinese Center for Disease Control and Prevention (Beijing, China).

\section{Patient consent for publication}

Not applicable.

\section{Competing interests}

The authors declare that they have no competing interests.

\section{References}

1. Prockop LD and Chichkova RI: Carbon monoxide intoxication: An updated review. J Neurol Sci 262: 122-130, 2007.

2. Hampson NB, Piantadosi CA, Thom SR and Weaver LK: Practice recommendations in the diagnosis, management, and prevention of carbon monoxide poisoning. Am J Respir Crit Care Med 186: 1095-1101, 2012.

3. Hampson NB and Weaver LK: Carbon monoxide poisoning: A new incidence for an old disease. Undersea Hyperb Med 34: 163-168, 2007.

4. Weaver LK: Hyperbaric oxygen therapy for carbon monoxide poisoning. Undersea Hyperb Med 41: 339-354, 2014.

5. Weaver LK, Hopkins RO, Chan KJ, Churchill S, Elliott CG, Clemmer TP, Orme JF Jr, Thomas FO and Morris AH: Hyperbaric oxygen for acute carbon monoxide poisoning. N Engl J Med 347: 1057-1067, 2002

6. Annane D, Chadda K, Gajdos P, Jars-Guincestre MC, Chevret S and Raphael JC: Hyperbaric oxygen therapy for acute domestic carbon monoxide poisoning: Two randomized controlled trials. Intensive Care Med 37: 486-492, 2011.

7. Xiang W, Xue H, Wang B, Li Y, Zhang J, Jiang C, Liang F, Pang $\mathrm{J}$ and $\mathrm{Yu} \mathrm{L}$ : Combined application of dexamethasone and hyperbaric oxygen therapy yields better efficacy for patients with delayed encephalopathy after acute carbon monoxide poisoning. Drug Des Devel Ther 11: 513-519, 2017.

8. Xiang W, Xue H, Wang B, Li Y, Zhang J, Jiang C and Pang J: Efficacy of N-butylphthalide and hyperbaric oxygen therapy on cognitive dysfunction in patients with delayed encephalopathy after acute carbon monoxide poisoning. Med Sci Monit 23: 1501-1506, 2017.

9. Goldstein M: Carbon monoxide poisoning. J Emerg Nurs 34: 538-542, 2008.

10. Guzman JA: Carbon monoxide poisoning. Crit Care Clin 28: 537-548, 2012

11. Hu H, Pan X, Wan Y, Zhang Q and Liang W: Factors affecting the prognosis of patients with delayed encephalopathy after acute carbon monoxide poisoning. Am J Emerg Med 29: 261-264, 2011.

12. Hou X, Ma L, Wu L, Zhang Y, Ge H, Li Z, Gao Y, Zhou Y and Gao C: Diffusion tensor imaging for predicting the clinical outcome of delayed encephalopathy of acute carbon monoxide poisoning. Eur Neurol 69: 275-280, 2013.

13. Weaver LK: Clinical practice. Carbon monoxide poisoning. N Engl J Med 360: 1217-1225, 2009.

14. Weaver LK, Valentine KJ and Hopkins RO: Carbon monoxide poisoning: risk factors for cognitive sequelae and the role of hyperbaric oxygen. Am J Respir Crit Care Med 176: 491-497, 2007.

15. Kudo K, Otsuka K, Yagi J, Sanjo K, Koizumi N, Koeda A, Umetsu MY, Yoshioka Y, Mizugai A, Mita T, et al: Predictors for delayed encephalopathy following acute carbon monoxide poisoning. BMC Emerg Med 14: 3, 2014.

16. Choi IS: Delayed neurologic sequelae in carbon monoxide intoxication. Arch Neurol 40: 433-435, 1983. 
17. Kim JH, Chang KH, Song IC, Kim KH, Kwon BJ, Kim HC, Kim JH and Han MH: Delayed encephalopathy of acute carbon monoxide intoxication: Diffusivity of cerebral white matter lesions. AJNR Am J Neuroradiol 24: 1592-1597, 2003.

18. O'Donnell P, Buxton PJ, Pitkin A and Jarvis LJ: The magnetic resonance imaging appearances of the brain in acute carbon monoxide poisoning. Clin Radiol 55: 273-280, 2000.

19. Hsiao CL, Kuo HC and Huang CC: Delayed encephalopathy after carbon monoxide intoxication-long-term prognosis and correlation of clinical manifestations and neuroimages. Acta Neurol Taiwan 13: 64-70, 2004.

20. Pavese N, Napolitano A, De Iaco G, Canapicchi R, Collavoli PL, Lucetti C, Gambaccini G and Bonuccelli U: Clinical outcome and magnetic resonance imaging of carbon monoxide intoxication. A long-term follow-up study. Ital J Neurol Sci 20: 171-178, 1999.

21. Choi IS, Kim SK, Choi YC, Lee SS and Lee MS: Evaluation of outcome after acute carbon monoxide poisoning by brain CT. J Korean Med Sci 8: 78-83, 1993.

22. Piantadosi CA: Diagnosis and treatment of carbon monoxide poisoning. Respir Care Clin N Am 5: 183-202, 1999.
23. Blumenthal I: Carbon monoxide poisoning. J R Soc Med 94: 270-272, 2001.

24. Knaus WA, Draper EA, Wagner DP and Zimmerman JE: APACHE II: A severity of disease classification system. Crit Care Med 13: 818-829, 1985.

25. Teasdale G and Jennett B: Assessment of coma and impaired consciousness. A practical scale. Lancet 2: 81-84, 1974.

26. Tombaugh TN and McIntyre NJ: The mini-mental state examination: A comprehensive review. J Am Geriatr Soc 40: 922-935, 1992.

(i) $\mathrm{T}$ This work is licensed under a Creative Commons Attribution-NonCommercial-NoDerivatives 4.0 International (CC BY-NC-ND 4.0) License. 